\title{
Dielectric relaxation in pulsed excimer laser ablated amorphous zirconium titanate thin films
}

\author{
$\underline{\text { P.Victor }}^{1}$ J. Nagaraju ${ }^{2}$ and S.B.Krupanidhi ${ }^{1}$ \\ ${ }^{1}$ Materials Research Center, Indian Institute of Science, Bangalore, India \\ ${ }^{2}$ Department of Instrumentation, Indian Institute of Science, Bangalore, India \\ victor@mrc.iisc.ernet.in
}

\begin{abstract}
Amorphous zirconium titanate thin films was prepared on $\mathrm{Pt}$ coated silicon substrates by pulsed excimer laser ablation technique at a very low substrate temperature of $200-300^{\circ} \mathrm{C}$ in $\mathrm{O}_{2}$ ambient. The temperature and frequency dispersion of dielectric permittivity was investigated on amorphous $\mathrm{ZrTiO}_{4}$ thin films. These films exhibited a marked dielectric relaxation at temperatures $500-700 \mathrm{~K}$ in a frequency range of $0.1-100 \mathrm{kHz}$. This behaviour was explained based on a dipolar relaxation. Analysis of the temperature dependence on relaxation time and electrical conductivity were done. An investigation on the correlation between mechanisms and models of the dielectric relaxation, origin of dielectric relaxation and the thermally activated motion of ionized defects in the amorphous structure are discussed.
\end{abstract}

\section{INTRODUCTION}

Scaling down of the chips toward smaller dimensions using traditional low dielectric constant materials is limited because of the electric fields, which eventually alters the device performance. Hence a serious issue has been considered on developing new materials with higher dielectric constant and lower leakage currents. Van dover et.al [1] has identified the amorphous $\mathrm{Zr}-\mathrm{Sn}-\mathrm{Ti}-\mathrm{O}$ is the most potential candidate for the DRAM applications [2] because of the higher dielectric constant, lower leakage currents and higher dielectric breakdown strength. $\mathrm{ZrTiO}_{4}$ and $\mathrm{Sn}$ modified $\mathrm{ZrTiO}_{4}$ thin films finds its extensive applications in the mobile communications inclusive of phase shifters, voltage tunable oscillators and filters.[3] They are widely used in the satellite communications because of its higher dielectric constant at the microwave frequency regime and almost zero temperature coefficient of resonance.

There had been recent trend in seeking the amorphous thin films over the crystalline thin films, as the electrical properties has a major impact in the degradation of the device and these crystalline thin films require higher temperatures for the crystallization which eventually destroys the microstructure of the thin films. Hence the amorphous thin films has become the major attraction for the MMIC and ULSI devices. There had been intensive work carried on the amorphous ferroelectrics and dielectrics in the early 1970's. $[4,5]$ In this work, we had analysed the dielectric relaxation phenomena in the amorphous $\mathrm{ZrTiO}_{4}$ thin films based on the dielectric dispersion of temperature and frequency.

\section{EXPERIMENT}

A dense ceramic $\mathrm{ZrTiO}_{4}$ target was prepared via conventional solid state reaction method by mixing, calcining the multi - component oxides at $1450^{\circ} \mathrm{C}$ for 6 hours and the powders was pressed into a $12 \mathrm{~mm}$ target. Single phase $\mathrm{ZrTiO}_{4}$ target was used for the laser ablation. $\mathrm{ZrTiO}_{4}$ thin films was deposited on pt(111) coated Si substrate by pulsed excimer laser ablation technique (Lambda Physik KrF, $248 \mathrm{~nm}$ ). [6] The base pressure of the deposition chamber was brought down to $10^{-6} \mathrm{mTorr}$ and $\mathrm{ZrTiO}_{4}$ thin films was deposited at an operating pressure of $10-25$ mtorr in an ultra pure oxygen environment. The deposition was carried at a laser fluence of $4 \mathrm{~J} / \mathrm{cm}^{2}$ and the substrate temperature varied from room temperature to $300^{\circ} \mathrm{C}$. The low temperature deposition was carried out intentionally, not to induce crystallinity in the thin films. The oxide film thickness was measured by optical spectrometer ( Filmetrics F20) and the $\mathrm{ZrTiO}_{4}$ thickness varied from $200-500 \mathrm{~nm}$. The structure, morphology and composition of the laser ablated $\mathrm{ZrTiO}_{4}$ thin films was characterized by $\mathrm{x}$ - ray diffraction (XRD), scanning electron microscope (SEM) and energy dispersive analysis spectroscopy (EDAX) respectively. The electrical characterization of the $\mathrm{ZrTiO}_{4}$ thin film capacitors was measured in MIM configuration. The gate electrodes of a circular area $1.96 \times 10^{-3} \mathrm{~cm}^{2}$ was fabricated by evaporating gold on to the thin films using a shadow mask for the formation of MIM capacitor. The MIM capacitor was used to study the capacitance measurements and the Keithley $3330 \mathrm{LCZ}$ meter to measure capacitances in the range from $40 \mathrm{~Hz}$ to $100 \mathrm{kHz}$. All the measurements were carried at elevated temperatures from $300-550 \mathrm{~K}$. 


\section{RESULTS AND DISCUSSION}

\section{Strucural Characterization}

$\mathrm{ZrTiO}_{4}$ target and the deposited thin films was characterized using $\mathrm{x}$ - ray diffraction (Scintag $\mathrm{Cu} \mathrm{K \alpha}$ ) for phase determination and the target reveals a single phase orthorhombic structure while the ZT thin films exhibits amorphous in nature as shown in figure 1 .

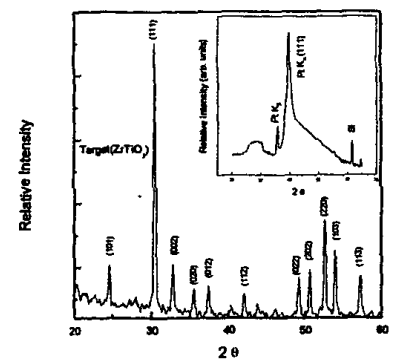

Fig. 1. X - ray diffraction of ZT target and thinfilms

\section{DC bias dependence}

The investigation of the dc bias dependence of the dielectric constant was carried out. The small signal capacitance was measured when the dc electric field was swept from negative bias to a positive bias and back again at a sinusoidal voltage of $0.5 \mathrm{~V}$ as shown in the figure 2. Both the $\tan \delta$ and the capacitance versus voltage exhibits non hysteresis behaviour. The figure 2 shows that the capacitance has very little influence on the sign of the applied fields and this may be considered the MIM configuration to be having ohmic contacts with the $\mathrm{ZrTiO}_{4}$ thin films.

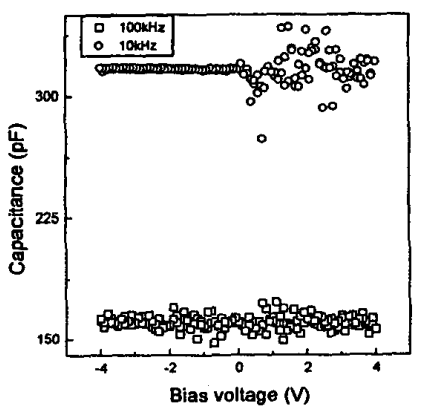

Fig. 2. Capacitance - voltage measurement of ZT thin films

\section{Dielectric measurements}

Information regarding the structural and electrical characteristics of the amorphous materials can be obtained from the dielectric relaxation measurements as the motion of ions mainly controls them.

\section{Frequency variation}

The variation of capacitance and $\tan \delta$ with frequency is shown in the figure 3 and 4 . In the figure 3, the capacitance exhibits larger dispersion at the low frequencies at higher temperatures, and is mainly attributed to the ionic space charges. [7]

This type of low frequency dispersion is exhibited either by pure debye or Maxwell - wagner relaxation mechanisms. A similar enhancement of the dielectric constant with the low frequency had been observed in the glassy materials and polymers which is due to the relaxation of the molecular dipoles. [8] The dispersion of $\tan \delta$ at low frequencies is exhibited and the dielectric loss peak moves to the lower frequency region as the temperature is decreased and attributed to the slowing down of ion motion. The loss peak is observed at the temperatures above $125^{\circ} \mathrm{C}$. This might be due to the onset of dc conduction or a loss peak appearing due additional ionic charge carrying
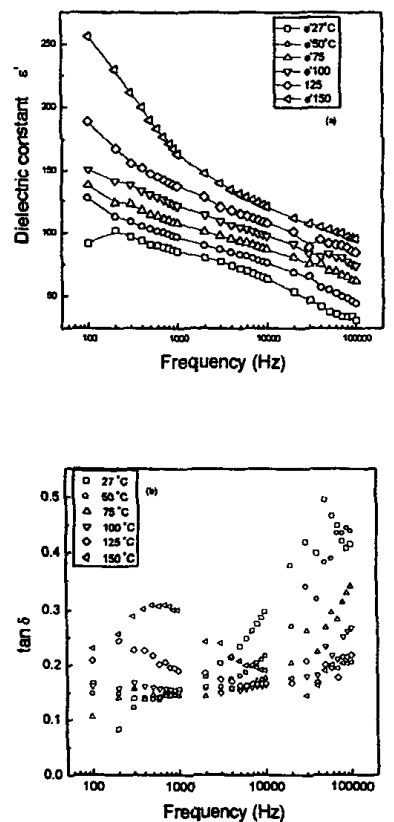

Fig. 3., a) dielectric constant versus frequency b) $\tan \delta$ versus frequency of MIM capacitor 
mechanism, which overshadows the high - frequency mechanism and it is also strongly temperature dependent. The dielectric constant was 34 for $100 \mathrm{kHz}$ at room temperature and the value increased as the measuring frequency decreased indicating dielectric relaxation.

The loss maxima is bserved at the changeover frequency in $\varepsilon^{1}-\omega$ plots for corresponding temperatures which is the consequence of Krammers - Kronig relation

$$
\chi^{\prime}(0)=\varepsilon^{l}(0)-1=\frac{2}{\pi} \int \chi^{\prime \prime}(\omega) d \ln (\omega)
$$

where $\chi^{\prime}(\omega)$ and $\chi^{\prime \prime}(\omega)$ represents the real and imaginary part of the frequency dependent complex dielectric response and the limits of the integration extend from $-\infty$ to $+\infty$.

The slope of $\varepsilon^{\prime}$ vs $\omega$ is 0.21 and it doesn't satisfy the debye relaxation as its slope is $\omega^{1}$ before the loss peak and $\omega^{-1}$ after the loss peak. The appearance of the loss peak indicates the dc conduction is not the process aiding, instead its of ionic process. There has been reports that the possibility of both the electronic and ionic, charge hopping processes participates in the low frequency dispersion.

The slopes of the loss curve at higher temperatures where prominent peaks are observed, the intial slope of the curve at the lower frequencies was proportional to $\omega^{-\mathrm{n}}$, with $\mathrm{n}$ varying from $0.5-0.65$ depending on the temperature. The slopes obtained before and after the maxima loss peak was 0.11 and 0.24 respectively which vividly shows that it doesn't obey the pure debye relaxation or the finite conductivity of the sample. On considering the two parallel RC elements being connected in series, the loss is initially dominated by the resistive part giving rise to a decrease of $\varepsilon^{\text {Il }}$ with frequency and at higher frequencies the capacitive part dominates. This is the other type of polarization, which is termed as the space - charge or interfacial polarization effects. This arises for heterogenous phases or at the interfaces of two materials with different conductivities inclusive of grain-boundary/grain , electrode/sample boundary and interphase boundaries. [9]

On considering a mixture of two materials in a condenser, the maxwell wagner relaxation model's real part is given by

$\varepsilon^{l}=\varepsilon_{\infty}^{M-W}+\left(\frac{\varepsilon_{s}^{M-W}-\varepsilon_{\infty}^{M-W}}{1+\omega^{2} \tau^{2}}\right)$

where $\quad(\omega \rightarrow 0) \varepsilon_{\infty}^{M-W}=\left(\frac{\tau_{1}+\tau_{2}-\tau}{C_{o}\left(R_{1}+R_{2}\right) \tau}\right)$ and

$(\omega \rightarrow \infty) \varepsilon_{\infty}^{M-W}=\left(\frac{\tau_{1} \tau_{2}}{C_{o}\left(R_{1}+R_{2}\right) \tau}\right)$ and $\tau_{1}, \tau_{2}$ are

the relaxation time and $R_{1}, R_{2}$ are the resistance of the two materials in the condenser. As the Maxwell wagner equation does not allow for a distribution of relaxation times, hence a modified equation with the Havriliak - Negami equation solves the purpose and is given as

$$
\varepsilon^{l}=\varepsilon_{\infty}^{M-W}+\left(\frac{\varepsilon_{s}^{M-W}-\varepsilon_{\infty}^{M-W}}{\left(1+\left(\omega^{2} \tau^{2}\right)^{(l-\alpha)}\right)^{\beta}}\right)
$$

The simulated above equation with the experimental curve agrees well for the $\alpha=0.25$ and $\beta=0.91$ which gives the measure of the relaxation times.

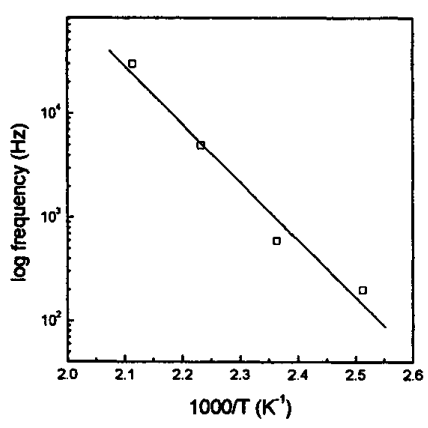

Fig. 4. Dielectric relaxation frequency versus $1 / \mathrm{T}$ of the $\mathrm{ZrTiO}_{4}$ thin films

The dielectric relaxation time can be calculated from the maxima of the dielectric loss peak and from the cole - cole plot of $\varepsilon^{1}-\varepsilon^{11}$ using $\omega \tau=1$. The additional temperature measurements are needed to trace the whole interfacial relaxation peak. The calculated activation energy from the Arhennius plot of relaxation frequency is $1.03 \mathrm{eV}$. This activation energy might be due to the migration of positively charged oxygen ion vacancies, which could have formed at the metal - oxide interface by an electrode reaction.

\section{DISCUSSION}

Amorphous zirconium titanate thin films exhibited temperature and frequency dispersion of permittivity. The obtained activation energy was higher and doesn't agree with Sakanavi type relaxation $\left(\mathrm{E}_{\mathrm{a}}=0.2-0.5 \mathrm{eV}\right)$, which is based on the thermal motions of $\mathrm{Ti}^{4+}$ ions between the potential minima within a distorted oxygen octahedra in crystalline materials.

The amorphous thin films lack long range periodicity and the presence of numerous defects will cause the tailing of band edges. These yields localized states to arise from broken titanium bonds associated with oxygen vacancies in amorphous $\mathrm{ZrTiO}_{4}$ thin films. The positive charge of the oxygen vacancy combination with other defects like $\mathrm{Ti}^{3+}$ can 
stabilize the complex $\left(\left(V_{o}^{\cdot *}-2 T i^{3+}\right)\right.$ by forming an electric and elastic dipole, which eventually leads to the dielectric relaxation under an alternating field. [10] This is due to the change in the orientation of the dipolar moment of the defect complex. The obtained activation energy of $1.04 \mathrm{eV}$ obeys well with the activation energy calculated from the diffusion of the doubly ionized oxygen vacancy in a $\mathrm{SrTiO}_{3}$ thin films $(0.98-1.1 \mathrm{eV})$. [11]

\section{CONCLUSION}

The amorphous $\mathrm{ZrTiO}_{4}$ thin films were prepared by laser ablation technique and the dielectric measurements were carried out at elevated temperatures. The modified Maxwell - Wagner with Havriliak Negami equation were found to fit in the experimental results. The activation energy obtained was due to the migration of positively charged oxygen ion vacancies formed by electrode reaction.

\section{ACKNOWLEDGEMENTS:}

One of the author (P.Victor) wishes to acknowledge CSIR, India for granting SRF graduate fellowship.

\section{REFERENCES:}

[1] R.B. Van dover, L.F.Schneemeyer and R.M.Fleming, "Discovery of a useful thin film dielectric using a compositional spread approach", nature 392, 162,1998

[2] E.S.Ramakrishnan, Kenneth D.Cornett, Gary H.Shapiro and Wei - Yean Howng, "Dielectric properties of radio frequency magnetron sputter deposited zirconium titanate based thin films", J. Electrochem. Soc., 145(1) , 358 (1998)

[3] K.Wakino, T.Nishikawa, Y.Ishikawa and H.Tamura, "Dielectric resonator materials and their applications for mobile communication systems" Br. Ceram. Trans. J. 89 (2), 39 (1990)

[4] C.J.Ridge, P.J. Harrop and D.S.Campbell., "Amorphous $\mathrm{Pb}-\mathrm{Ti}-\mathrm{O}$ capacitors", Thin solid Films., 2, 413,1968

[5] Tsuya and K.Arai., " Amorphous ribbons of $\mathrm{LiNbO}_{3}$, $\mathrm{PbTiO}_{3}$ and $\left(\mathrm{PbTiO}_{3}\right)_{0.4}\left(\mathrm{~B}_{2} \mathrm{O}_{3}\right)_{0.1}$ " Jpn. J. Appl. Phys. $18,461,1979$

[6] P.Victor, J.Nagaraju and S.B.Krupanidhi.,"Growth and electrical properties of highly oriented $\mathrm{ZrTiO}_{4}$ thin films", Thin solid films , communicated 2002

[7] Sudipta Bhattacharya, " Aurivillus type Bismuth layered perovskite thin films by laser ablation for NVRAM applications"., Ph.D thesis, Indian Institute of Science, Bangalore, India, 2001.

[8] J.M.Stevels, " The electrical properties of glass", Handbuch der physik, vol. 20. Berlin : Springer Verlag, 1957, pp. 350-391.
[9] A.R.Von Hippel, “ Dielectric and waves", Wiley, New York, 1954.

[10] R.Lohkamper, H.Neuman, and G.Arlt, “ Internal bias in acceptor doped $\mathrm{BaTiO}_{3}$ ceramics: numerical evaluation of increase and decrease", J. Appl. Phys. 68, 4220, 1990

[11] k.Morii, H.Kawano, I.Fujii, T.Matsui and Y.Nakayama., “ Dielectric relaxation in amorphous thin films of $\mathrm{SrTiO}_{3}$ at elevated temperatures", J. Appl. Phys. 78 (3), 1914, 1995 